\title{
An integrative analysis of meningioma tumors reveals the determinant genes and pathways of malignant transformation
}

\author{
José Carlos Iglesias Gómez ${ }^{1}$ and Adrián Mosquera Orgueira ${ }^{2}$ * \\ 1 Independent Video Editor, Santiago de Compostela, Spain \\ 2 Independent Researcher, A Coruña, Spain
}

Edited by:

Sven Bilke, National Institutes of Health, USA

\section{Reviewed by:}

Paola Parrella, I.R.C.C.S. Casa Sollievo della Sofferenza, Italy

Sven Bilke, National Institutes of Health, USA

\section{*Correspondence:}

Adrián Mosquera Orgueira,

Independent Researcher, Mesón do

Vento No. 56, Ordes, A Coruña 15689,

Spain

e-mail: adrian.mosquera@live.com
Meningiomas are frequent central nervous system neoplasms, which despite their predominant benignity, show sporadically malignant behavior. Type 2 neurofibromatosis and polymorphisms in several genes have been associated with meningioma risk and are probably involved in its pathogenesis. Although GWAS studies have found loci related to meningioma risk, little is known about the factors determining malignant transformation. Thus, this study is aimed to identify the genomic and transcriptomic factors influencing evolution from benignity toward aggressive phenotypes. By applying an integrative bioinformatics pipeline combining public information on a wealth of biological layers of complexity (from genetic polymorphisms to protein interactions), this study identified a module of co-expressed genes highly correlated with tumor stage and statistically linked to several genomic regions (module Quantitative Trait Loci, mQTLs). Ontology analysis of the transcription hub genes identified microtubule-associated cell-cycle processes as key drivers of such network. mQTLs and single nucleotide polymorphisms associated with meningioma stage were replicated in an alternative meningioma cohort, and integration of these results with up-to-date scientific literature and several databases retrieved a list of genes and pathways with a potentially important role in meningioma malignancy. As a result, cytoskeleton and cell-cell adhesion pathways, calcium-channels and glutamate receptors, as well as oxidoreductase and endoplasmic reticulum-associated degradation pathways were found to be the most important and redundant findings associated to meningioma progression. This study presents an integrated view of the pathways involved in meningioma malignant conversion and paves the way for the development of new research lines that will improve our understanding of meningioma biology.

Keywords: meningioma, cancer, whole-genome, integrative analysis, transcriptomics, malignant conversion, video presentation, no funding research

\section{INTRODUCTION}

Meningiomas represent approximately a quarter of the total central nervous system (CNS) neoplasms. These tumors are derived from normal arachnoidal cells of the leptomeninges, appear tightly joined to the Dura Mater, and tend to be located along the parasagital sinus, over the cerebral convexity, in the sphenoid wing, around the pontocerebellar angle or along the dorsal region of the spinal cord. Although as a group they are considered to be benign, variability in recurrence frequency, life expectancy prognosis, symptoms, and histological appearance exists. In this regard, histological analysis reveals that $80-90 \%$ of the meningiomas are benign [World Health Organization (WHO) Grade I], which are not associated with an excess of mortality when totally resected. However, about $5-15 \%$ of them are atypical (WHO Grade II) and associated with a marked increase in recurrence frequency and a small risk of death. Only $1-3 \%$ of the cases become anaplasic or malignant (WHO Grade III), developing a high tendency to invade brain structures, metastasize, and to recur (1-3). Overall, Grade II tumors have an average life expectancy of 11.9 years and an average recurrence-free survival of 142.5 months vs. 3.3 years of average life expectancy and 39.8 months of recurrence-free survival for Grade III meningiomas (4).

Ionizing radiation exposure is the principal modifiable risk factor. Age and female gender (especially during the reproductive period) are known non-modifiable risk factors. Nevertheless, genetic factors have also been found to play a role in meningioma development and predisposition. Type 2 neurofibromatosis (NF2) is an autosomal dominant condition related to a mutation on chromosome 22q12 and is a common condition related to elevated risk for developing meningiomas, among other neoplasms (4). In fact, loss of heterozygosity at several points in the 22q locus has been shown to be an early event in the development of benign meningiomas. Curiously, a study by Black et al. (5) revealed that meningioma gene expression is correlated with progesterone receptor levels, and this is particularly true for those genes encoded near 22q12. Single nucleotide polymorphisms (SNPs) in the Ki-RAS and $E R C C 2$ genes were associated with increased risk for developing meningiomas. Genes involved in detoxification, reactive 


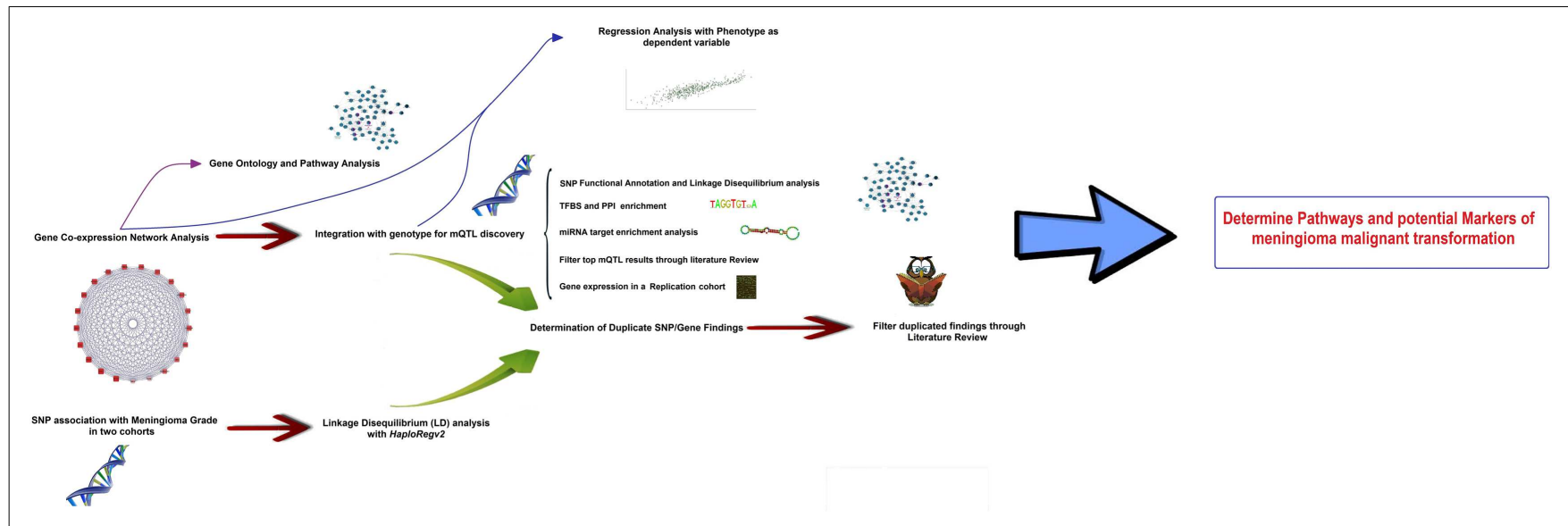

FIGURE 1 | Pipeline scheme representing all the steps followed in this research

oxygen species mitigation, metabolism, and DNA-repair also seem to be involved. In this regard, SNPs in the $\mathrm{C}$ variant of SOD3 (superoxide dismutase 3), GSTT1 (glutathione $S$-transferase theta 1), and MUTYH (Muty homolog) have been found to be associated with meningioma risk. Moreover, association of SNPs located in genes related to apoptosis (CASP8) and cell-cycle pathways (GLTSCR1, ERCC4, and PCNA) are also known (1).

Since the discovery of the scale-free property back on 1999, living phenomena have been shown to be organized in networks regulated by this property (6-14). Hovarth et al. (15) have shown that gene expression can be analyzed as partially discrete coexpression networks where connectivity among genes follows the scale-free property. The main aim of this study was to assess the relationship between genomic markers and gene-expression data of meningioma tumors at the network level. The results of this analysis were integrated with protein-protein interaction (PPI) networks, transcription factor binding sites (TFBS), miRNA target sites, and pathways data. Two previous gene-expression analyses and a genotypic study were used for replication purposes. Overall, redundant findings for the implication of several pathways in meningioma progression are described, and a list of potential markers is provided for future experimental validation.

\section{MATERIALS AND METHODS}

The core of the study is based on the integration of genomic marker information (i.e., SNPs) with gene-expression data on a group of meningioma tumors, published by Nelson et al. (16), in the search for genomic loci influencing malignant-like gene expression. In the first part, Weighted Gene Co-expression Network Analysis, WGCNA $(15,17)$ was applied to explore gene expression as an undirected coexpression network and reduce its dimensionality. A co-expression module deeply correlated with meningioma biological parameters was discovered, and the top hub genes inside the module were identified based on network analysis parameters. In a second step, genetic loci associated to the meningioma-related co-expression network were identified, in an approach known as module Quantitative Trait Loci [mQTLs (18)]. These loci were found to partially overlap with SNP association with disease stage. In the third step, the most likely causative genes in the proximity of the mQTLs were delimited, which formed the input list for their integration with PPI networks, TFBS data, miRNA signatures, and pathways databases. Multivariate regression models were created in order to determine what extent of the variability in WHO meningioma Grade could be explained by mQTL SNPs and co-expression module data. With the help of literature filtering, a list of genes with a high potential role in meningioma malignant conversion is provided for future experimental testing. A study pipeline scheme can be consulted in Figure 1.

\section{INITIAL DATA SOURCE}

The initial data input for this study was of a group of 85 meningioma samples [GEO accession GSE16584 (16)]. Meningioma samples ( 22 males and 37 females) with both genotypic and geneexpression data were selected. This resulted in a total number of 59 samples, of which 39 were WHO Grade I meningioma cases, 15 were WHO Grade II, and 5 were WHO Grade III (Table S1 in Supplementary Material). Tumor genotyping was performed with the Human Mapping 100K Set platform (Affymetrix) and geneexpression data were obtained using GeneChip Human Genome U133 Plus 2.0 Arrays (Affymetrix).

Genotyping Hind and Xba CEL files were downloaded from GEO [Gene-Expression Omnibus $(19,20)]$; accession code GSE16583 (16) and analyzed the Affymetrix Genotyping Console software (Affymetrix). Only samples with a quality control $\geq 90 \%$ were included. After pre-processing all the data results, we selected 111,829 SNPs, which passed three filters: minimum allele frequency of $5 \%$, minimum call rate of $95 \%$, and evidence of Hardy-Weinberg equilibrium $(P$-value $\geq 0.01)$.

\section{GENE-EXPRESSION ANALYSIS}

Expression CEL files were also downloaded from GEO (GEO accession code GSE16581) and analyzed in $R(21)$. Samples were read, background corrected, normalized, probe-specific background corrected, and summarized into an $R$ eset class object using functions of the affy package (22). Array quality was determined with the arrayQualityMetrics package (23). In order to simplify the analysis, we applied a filter to select only those roughly 12,000 
probes, which showed at least a 1.8-fold expression change according to the median in at least $10 \%$ of the samples (24). In order to find expression quantitative trait loci (eQTLs), and since no Y chromosome SNPs were measured in the chips, we also excluded gene-expression probes pertaining to such location.

Weighted gene co-expression network analysis functions were applied to expression data according to several online tutorials (18). The adjacency matrix was calculated using a softthresholding power of 6 , which showed an approximate scalefree topology $\left(R^{2}=0.75\right)$. Briefly, the connectivity value of each selected transcript (calculated similarly to kIN in Section "Regression Models") was used to create a group of 10 bins with equal size, and each connectivity value was assigned to each bin. The connectivity distribution $k$ was defined as the average connectivity value for each bin, whilst the probability distribution of $k$ $p(k)$ was defined as the ratio of the number of connectivity values in each bin by the number of connectivity values studied. Under the approximate scale-free topology assumption, the logarithm of $p(k)(\log (p(k)))$ and the logarithm of $k(\log (k))$ are strongly negatively correlated. In this case, by using a soft-thresholding power of 6 we obtained a $R^{2}=0.75$ (Pearson's correlation of 0.86 , regression slope of -1.46), whilst using no thresholding at all we obtained $R^{2}=0.03$ (Pearson's correlation of 0.17 , regression slope of 1.05). Thus, the soft-thresholding value selected ensures approximated scale-free topology whilst retaining a higher number of informative connections in the network. Several co-expression modules were determined, and correlation between phenotypic data and their respective first principal components (a.k.a. module eigenge$n e s, \mathrm{ME}$ ) was calculated. Mean gene significance with WHO Grade was calculated as the absolute average correlation of all module genes with this trait. Due to its marked positive correlation with several parameters (refer to Figure 5), the pink-module was chosen for further analysis. Module membership (MM, a.k.a kME) was defined as the Spearman's correlation between the ME and the genes corresponding to the pink-module, which is considered a measure of centrality of each gene in the network. Gene-expression standard deviation was determined with the function rowS $d$, part of the package matrixStats (25).

\section{GENE CO-EXPRESSION NETWORK VISUALIZATION AND PATHWAY ANALYSIS}

Cytoscape (26) was used to create a graphic representation of the pink-module. Using the CentiScape plug-in (27), we analyzed several network parameters, such as degree and betweenness centrality. Hub genes were chosen as those with a degree higher or equal to 250 and a betweenness centrality value higher or equal to 2,500 . The ClueGO plug-in (28) was utilized to create pathway and Gene Ontology (29) enrichment networks. Gene ontology databases for Biological Processes, Cellular Components, Immune System Processes, and Molecular Functions, as well as $\operatorname{KEGG}(30,31)$ and Reactome (32), were included in the analysis. Enrichment analysis was performed with the hypergeometric test, and a significant FDR-adjusted $P$-value threshold of 0.001 was selected. ClueGO gene ontology and pathway terms were filtered, so that only those matching to at least $10 \%$ of the genes in the pink-module were considered. Gene ontology analysis for hub genes was performed with DAVID web tool $(33,34)$.

\section{mOTL FINDING, ANNOTATION, AND DOWNSTREAM FUNCTIONAL ANALYSIS}

The degree of association between the the pink's module ME and the genotypes was performed by fitting a logistic regression with SNPs as dependent phenotypes with the function spn.lhs.tests implemented in the package snpStats (35). Since the ME does not reflect all the variability of the whole module, the sum of the first and second principal components $(P C 1+P C 2)$ was also studied in a similar way. Finally, we also tested for significant improvements over the base model of the ME by separately adding PC2 and PC1 + PC2. SNPs with a $P$-value in the order of $10^{-5}$ or less were selected as mQTLs, and in case that various SNPs mapped to the same gene only the most significant was chosen.

mQTL putative genes were obtained from the HaploReg v2 web browser (36). mQTL enrichment in TFBS was studied using oPPOSUM Single Site Analysis tool (37). Briefly, the analysis was run with the following parameters: (1) Vertebrate JASPAR CORE profiles were selected, (2) only the top $10 \%$ conserved regions (with a minimum conservation of $70 \%$ ) between the mouse and the human genome were included with (3) a position weight matrix match threshold of $80 \%$ and (4) within a region of 5,000 bp up and downstream of the transcription start site for each gene.

Gene Ontology and Pathways analysis for mQTL putative genes was performed with Cytoscape and ClueGo similarly to Section "Gene Co-Expression Network Visualization and Pathway Analysis." In this case, a threshold of $3 \%$ was set to the proportion matching genes in the ontology group. Gene set enrichment analysis (GSEA) was used for miRNA target site enrichment (38). The PPI database STRING (39) was used to test for known interactions among the proteins encoded by the MQTL genes.

To address the possible influence of genes in the pink coexpression module over mQTLs, a search for mQTL putative genes matching to in-module genes was conducted. Finally, the PubMed database was interrogated for known associations between mQTL putative genes and cancer or meningioma (search terms: "Gene Name AND Cancer" and "Gene Name AND Meningioma", date: 28/4/2014).

\section{GENE EXPRESSION AND GENOTYPIC REPLICATION COHORTS}

An expression replication cohort was constructed with data from GEO [GEO accession codes GSE43290 (40) and GSE4780 (41)]. Matching samples in both datasets were selected, and batches were removed with the ComBat function implemented in the package sva (42), getting an overall cohort of 22,283 probes and 103 individuals. Linear models of the limma package (43) were applied to determine differential gene expression between tumor stages and to detect matches with pink-module genes. Wilcoxon Rank Sum Test was applied to determine possible connectivity differences among those probes found to be overexpressed in the replication cohort compared to its background. Background connectivity was determined as 10,000 times random permutation of $\mathrm{kME}$ values for pink-module probes present in the replication cohort.

At the same time, a genotypic cohort of 50 meningioma samples was downloaded from GEO [GEO accession code GSE42624 (44)], which was used to find overlapping disease progressionassociated SNPs/genes in both datasets, as well as to detect matches with mQTLs. Gene overlaps between the two genotypic cohorts 
and mQTLs were computed by including putative gene symbol matches for SNPs at a linkage disequilibrium value at least of 0.8 in individuals of European Ancestry included in the 1000 Genomes Project, according to the HaploReg web tool (36). Finally, literature analysis was performed for duplicate findings (e.g., SNPs related to the same putative gene in at least two of the three lists: stringent mQTL list and any of the two SNP association lists) as described for mQTLs above.

\section{REGRESSION MODELS}

Multivariate regression models with genotypic and transcriptomic data were regressed on phenotypic information. A vector was created containing the Spearman's correlation of the WHO Meningioma Grade distribution with each module probe, hereafter called Gene Significance for WHO Meningioma Grade (GS WHO_Grade). A analogous vector containing the Spearman's correlation of each module probe with each mQTL, was designated GSmQTL. Intramodular connectivity (kIN) was defined as the sum of the absolute value of the Spearman's correlation of each probe with all the other probes in the same module. Another measure of connectivity, known as kME or MM, was calculated as previously defined in Section "Gene-Expression Analysis."

Regression analysis was performed with the $l m$ function, implemented in the package stats (21). A stepwise selection of models based on Akaike information criterion (AIC) was conducted. Significant variables were selected for further analysis if their $P$-value in the best AIC model was in the order of $10^{-3}$ or below, according to a $T$ test. Rank transformation into normality function [rntransform, package GenABEL (45)] was applied to the dependent variables of the models. Normality of the dependent variables and the residuals was assessed with the function shapiro.test (package stats). Variance inflation factors (VIFs) for lineal models were calculated with the VIF function implemented in the package fmsb (46).

\section{MISCELLANEOUS}

Boxplots present in Figure 3C, Figures S1A and S2E in Supplementary Material were created with the "boxplot" function implemented in the graphics package (21), and they represent the upper and lower quartiles (upper and lower of the boxes), the median (horizontal line inside the boxes), as well as the most extreme values in the dataset (the whiskers and the points outside it). Note that the whiskers include dispersed data that fall within 1.5 times the height of the box, and points represent values lying outside this range. Expression missing values were imputed with functions of the package impute (47).

\section{RESULTS}

\section{CO-EXPRESSION ANALYSIS}

Weighted gene co-expression network analysis identified 16 coexpression modules. Module-trait relationships revealed that the pink ME was highly and significantly correlated with WHO Meningioma Grade, recurrence frequency, observed recurrence after sample, Ki-67 staining, and with the total sum of chromosome arm losses (Figure 2). Boxplots of mean gene significance with WHO_Grade revealed that the Pink-module contains the most correlated genes (Figure S1A in Supplementary Material). $M M$ (a.k.a kME) to the sixth power was determined to be in a marked 0.88 Spearman's correlation $\left(P\right.$-value $\left.<10^{-16}\right)$ with intramodular connectivity $(k I N)$, which is indicative of its usefulness to study high-level modular network properties. MM vs. GS WHO_Grade (Figure S1B in Supplementary Material) revealed

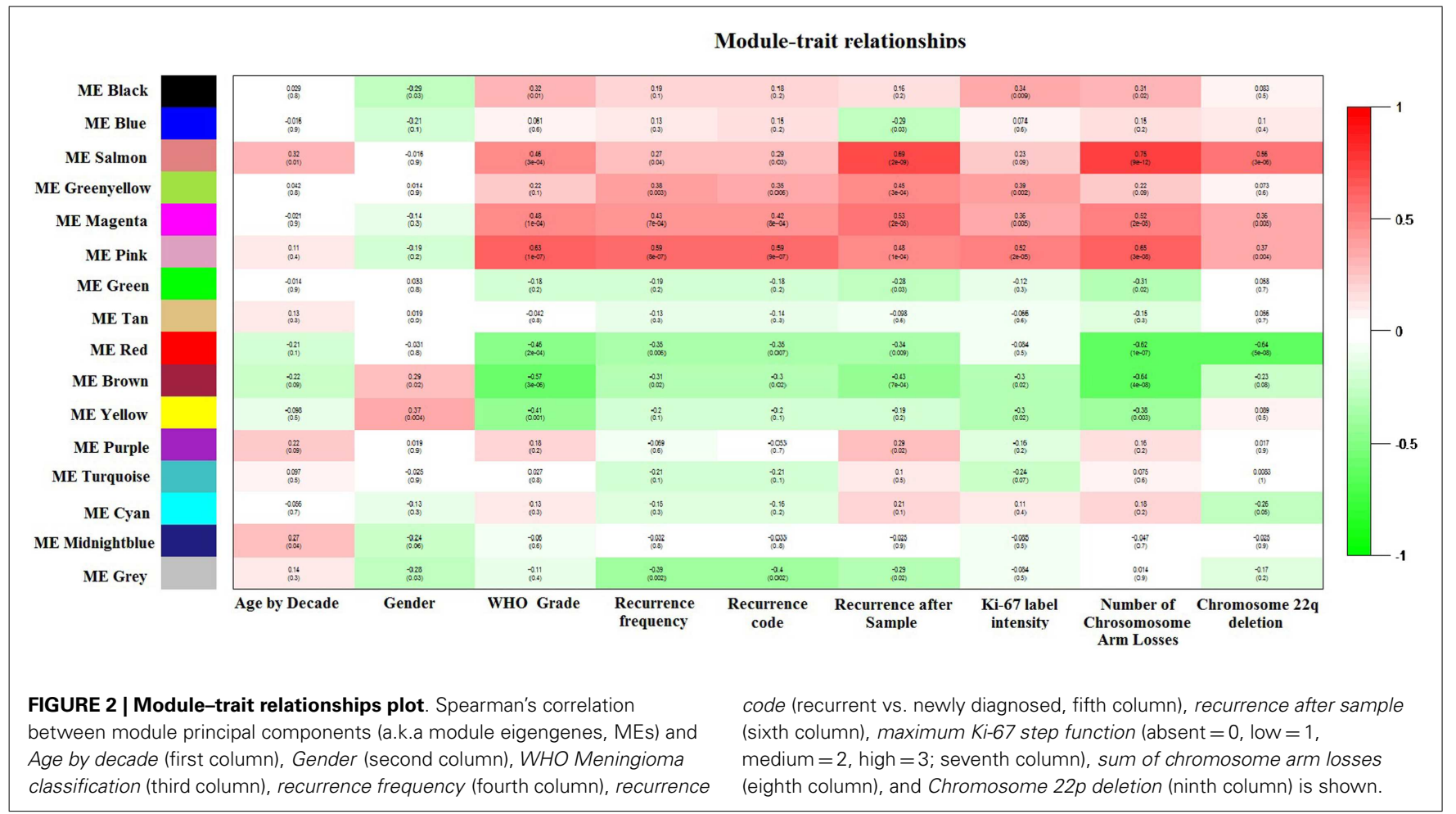




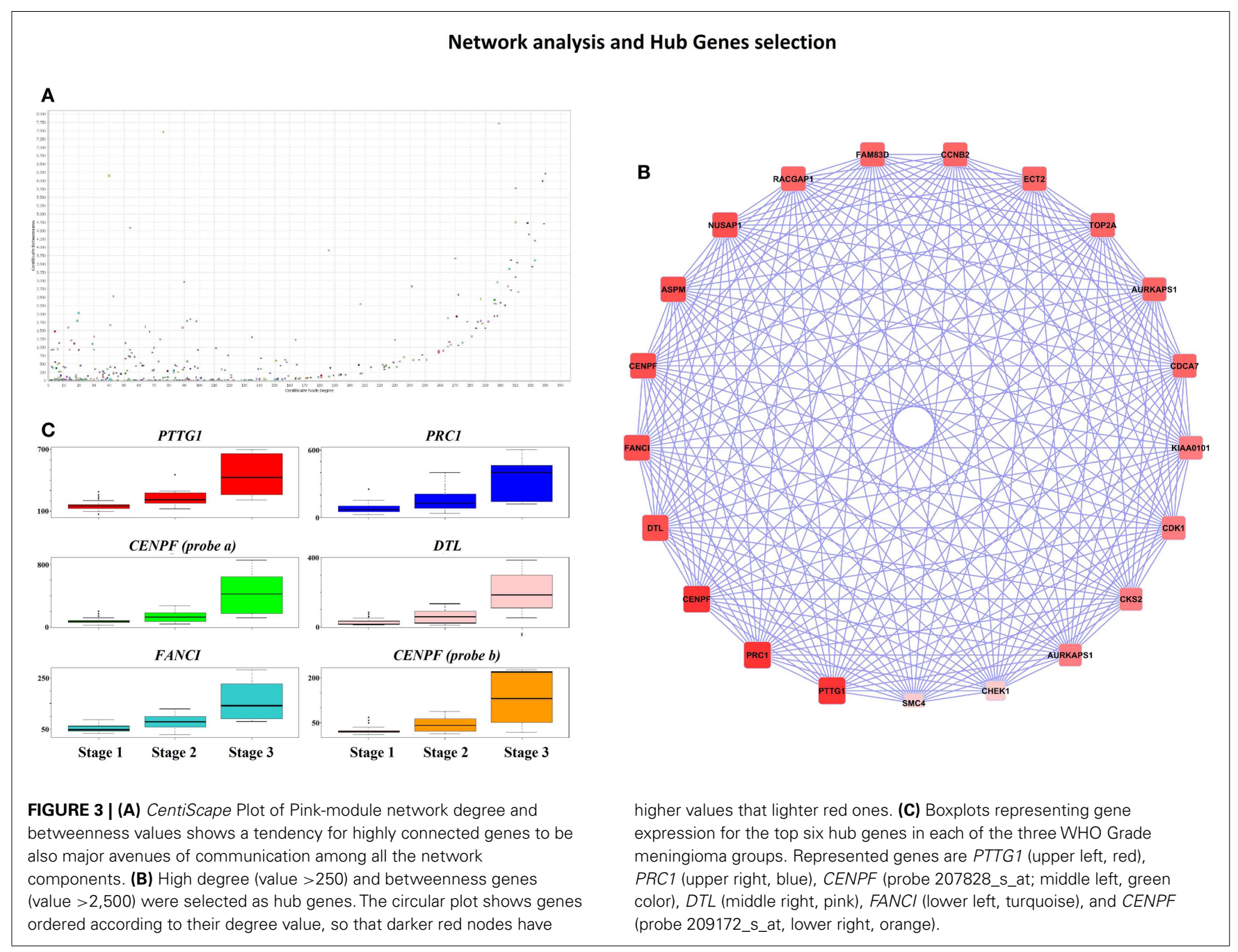

a 0.72 Spearman's correlation value $\left(P\right.$-value $\left.\leq 10^{-16}\right)$, showing that genes importantly associated with disease stage are also the more relevant in the module. Pink-module gene-expression standard deviation was found to be inversely correlated with kIN (Spearman's $r h o=-0.06$, one-sided $P$-value $=0.09$ ), but curiously higher levels of expression variability co-exist with low levels of connectivity (Figure S1C in Supplementary Material). For example, genes with a kIN below 2 are significantly more variable than those whose kIN value is above 2 (Wilcoxon Rank Sum Test $P$-value $=0.002$, 95\% C.I. $=7.74-36.96)$. This is consistent with the role of hub genes as central players in complex biological phenotypes (48). Moreover, almost 76\% of the pinkmodule genes found in the replication cohort were significantly differentially expressed between tumor stages (FDR-adjusted $P$ value $<0.05$ ), and differentially expressed genes had a significantly greater connectivity (MM or kME) than the overall group of pinkmodule genes (Wilcoxon Rank Sum Test $P$-value $=0.043$, 95\% C.I. $\left.=6.54 \times 10^{-5}-3.70 \times 10^{-2}\right)$.

Cytoscape analysis of the network was used to choose the hub genes based on their degree and betweenness values in the network (Figure 3A). All hub genes were positively correlated with WHO_Stage (Figure 3B and in Table S4 in Supplementary Material), and graphical information about the top six can be consulted in Figure 3C. Furthermore, a stringent search for Gene Ontology and Pathway terms enrichment using ClueGO revealed a marked predominance in cell-cycle pathways. Isoprenoid metabolic processes, intermediate filament bundle assembly, proteoglycan binding, and oxidoreductase activity terms also passed the cutoff (Figures 4A,B). DAVID ontology analysis for hub genes revealed a marked enrichment in processes related to microtubule cytoskeleton. This is remarkably true for spindle and chromosome-related microtubular processes (Table S5 in Supplementary Material).

\section{DETERMINATION OF mOTLS}

Genomic markers and gene-expression data were merged into a single study to analyze quantitative relationships between them (QTLs). Due to the established relationships between several meningioma characteristics and the genes in the Pink-Module, we sought to determine genetic loci particularly affecting its expression ( $m Q T L s$, Figure 5). By fitting logistic regression models, we found 48 SNPs associated with the ME at $P$-values in the range of $10^{-5}$ or less. Four of these had $P$-values in the range of $10^{-6}$ and 


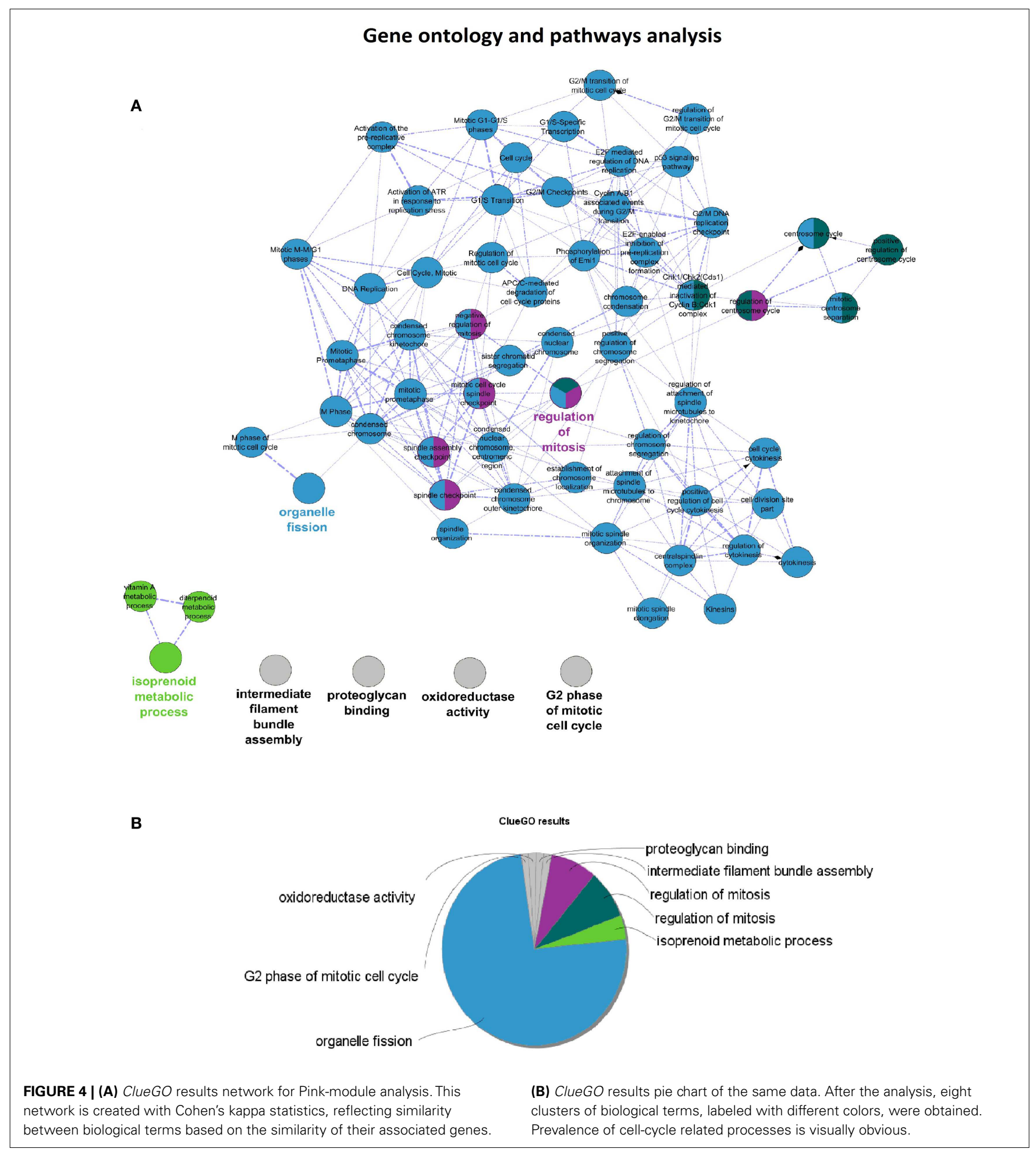

one was on the range of $10^{-7}$ (Table S2A in Supplementary Material). These SNPs correspond to four genomic loci mapping to the ANKRD50, LOC389705, NRP1, and HOXC13 genes. To test possible improvements to this model, we performed logistic regression where the ME was included in the base model and significance for association with PC2 and PC1 + PC2 was tested (Tables S2B,C in
Supplementary Material). The results indicated at an SNP in the GRIN3A gene with a $P$-value of roughly 0 , and others at the IBTK, $A N G P T 1, A S T N 2$, and LPIN2 genes with $P$-value in the range of $10^{-6}$ or below. Furthermore, logistic regression with PC1 + PC2 as regressor discovered 59 SNPs in 52 different genes showed $P$ values in the range of $10^{-5}$ or less (Table S2D in Supplementary 


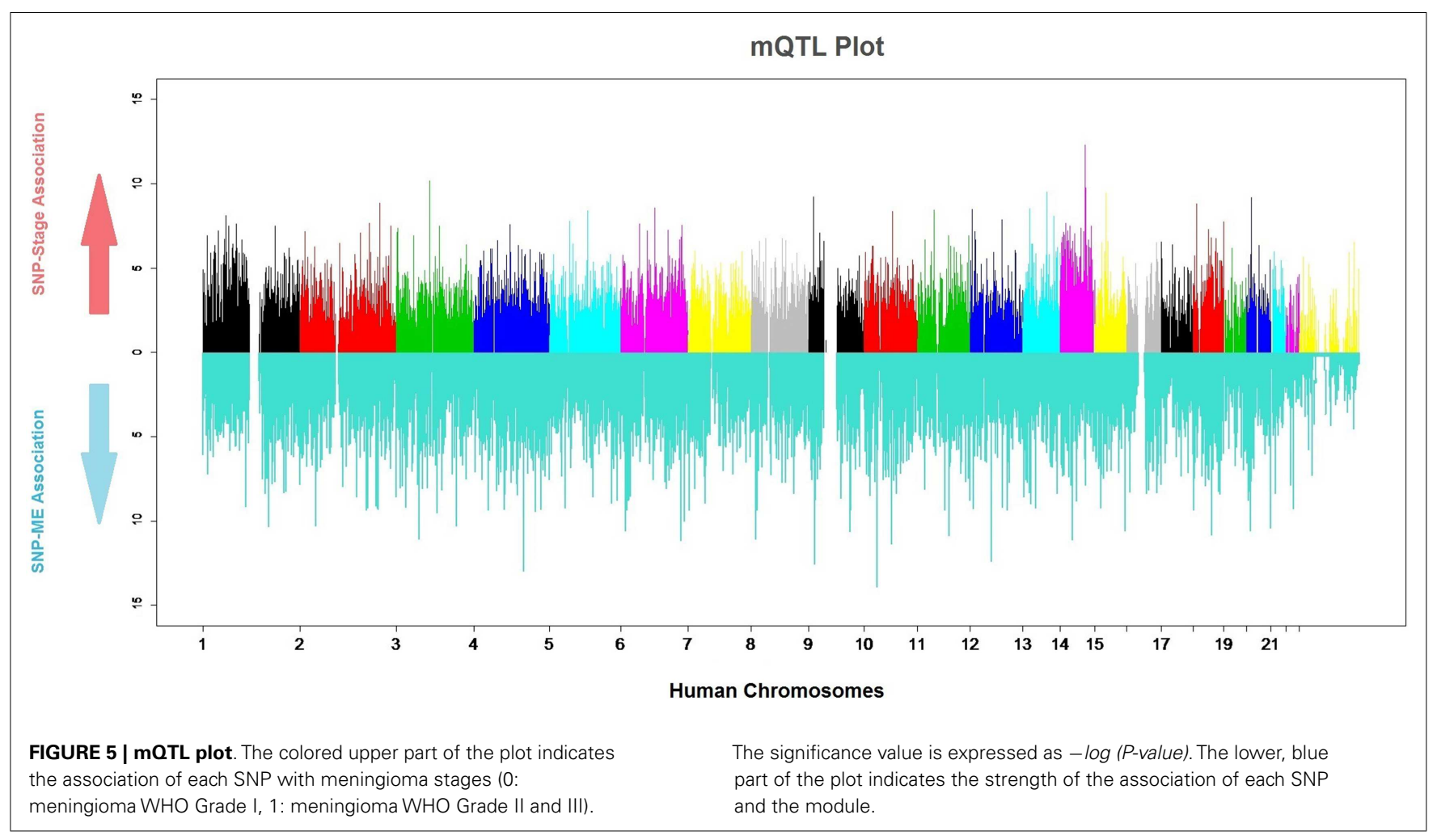

Material). Nine of the SNPs had $P$-values in the range of $10^{-6}$ and were located at or near to LOC339535, FMN2, NEK7, IL6R, C14orf64, KRT72, and SPRY2. Five of them had $P$-values in the range of $10^{-7}$ and were located at or near to ATF6, LOC100288079, USH2A, SUCLG2, and PHC1. Finally, eight SNPs were on the range of $10^{-8}$ mapping in or close to DNAJC19, HSP90AA4P, CHD10, XKR6, CA1, RASSF9, APAF1, and LOC100130792.

We made a search in PubMed for the known implication of all putative $\mathrm{mQTL}$ genes in cancer and meningioma. More than half of the genes ( 63 out of $108,58 \%$ ) have supporting evidence for their direct implication in cancer, whilst only 5 out of $108(5 \%)$ are known to be involved in meningioma pathogenesis. This difference is not surprising, since meningioma constitutes a less intensive research field than other cancers. The latter genes are ALCAM, NRP1, IGF1R, CA1, and ERCC4. Of these, NRP1 and IGF1R do not have a clearly related role in meningioma yet, but evidence points toward the implication of the former in meningiomaassociated neoangiogenesis (49) and for the blocking of the latter as therapeutic option (50).

GENE ONTOLOGY, PATHWAY, PROTEIN-PROTEIN INTERACTION, miRNA TARGET SITE, AND TFBS ENRICHMENT ANALYSIS OF mOTL GENES

A search for miRNA sets enriched in $m Q T L$ proximal genes was performed using GSEA, which revealed a significant enrichment in 36 of the sets (FDR-adjusted $P$-value $<0.05$ ). The two top findings were $M I R-124 A$ and $M I R-34 B$, both with FDR-adjusted $P$-values in the order of $10^{-5}$ (Table S2E in Supplementary Material).

Enrichment for gene ontology terms and pathways associated with mQTL genes using ClueGo revealed enrichment in adherens junction interactions and cell-cell junction organization, response to low-density lipoproteins and cholesterol storage, endothelial cell proliferation, sprouting angiogenesis, negative regulation of vascular permeability, calcium-channel activity, NMDA glutamate receptors, actin and myosin-related pathways, meiotic chromosome segregation, protein localization to mitochondrion, and oxidoreductase activity (Table S2F in Supplementary Material). All terms above showed FDR-adjusted $P$-values $<0.05$.

STRING database was used to detect known interactions between all putative $m Q T L$ genes at the PPI level. Using a high confidence interaction score, 9 interactions were detected between the 87 proteins present in the database. Only 2.96 interactions were expected under the null hypothesis, thus concluding that the mQTL PPI network is significantly enriched in interactions $(P$-value $<0.0035)$. Five of the interactions were among a cluster of four cadherin proteins (namely $\mathrm{CDH} 12, \mathrm{CDH} 13, \mathrm{CDH} 8$, and CDH9).

Transcription factor binding site (TFBS) enrichment was performed by including all mQTL putative genes in a search with oPPOSUM Human Single Site Analysis web tool. A marked significant enrichment in the Forkhead group of TFBS (FOXD3, FOXF2, FOXI1, FOXQ1, FOXA2, FOXD1), and in the Homeo group (LHX3, NOBOX, PDX1, PRRX2, NKX2-5, NKX3-1, and PBX1) was observed (Table S2G in Supplementary Material).

\section{SNP ASSOCIATION WITH TUMOR STAGE}

Tests for association between SNPs and tumor stage encoded as a binomial factor [either lowly malignant (stage I) or malignant (stages II and III)] on 84 subjects resulted in 5 SNPs ranking with a $P$-value on the order of $10^{-05}$ and 2 on the order of $10^{-06}$ (Figure 5; Table S2H in Supplementary Material). These two of 
the SNPs were located in an intergenic region $600 \mathrm{~kb} 5^{\prime}$ to SEL1L (rs1652605 and $r s 1958666)$. By applying the same method in a Spanish cohort to find duplicate meningioma stage associated regions, other six SNPs in the same intergenic region ( $r s 17669975$, $r s 11159518, r s 1198035, r s 1210840, r s 1953408$, and $r s 1198030)$ were also substantially associated with meningioma stage (Table S6 in Supplementary Material). Curiously, this region encodes a long non-coding RNA known as RP11-666E17.1-001, whose function is unknown.

Inflation values $(\lambda)(51)$ were estimated to be 1.15 for the first cohort and 1.02 for the second (replication) cohort.

\section{IN-MODULE GENES AND mOTL PUTATIVE GENES}

In the search for possible in-module genes that may play an important regulatory role at the genomic scale, we searched for in-module genes that mapped to any of the mQTL genes. BTBD3, LOC339535, and TBC1D9 genes were present in both lists, and thus are potentially important in meningioma pathogenesis.

\section{DUPLICATE SNP-RELATED GENE FINDINGS}

In addition to the SEL1L-related SNPs that we described above, we also found coincidences between other mQTL-related genes and the tumor stage association cohorts. By searching for SNPs related to the input genes in LD as described in the Section "Materials and Methods," we could identify SPRY2 as a gene with SNPs in strong linkage disequilibrium between the MQTL list and the first genotypic cohort, whilst overlaps were found between the mQTL list and the validation genotypic cohort at the C3orf67, C6, MEOX2, and SLC39A10 loci.

\section{MULTIVARIATE REGRESSION ANALYSIS}

Pink-module co-expression, mQTL, and meningioma phenotypic data were used to create multivariate regression models. In the first, $1 \mathrm{kME}$ was regressed on GS WHO_Grade (GS WHO_Grade $\sim k M E)$, and an adjusted $R^{2}$ value of 0.51 was retrieved $\left(P\right.$-value $<2.2 \times 10^{-16}, \mathrm{VIF}=2.04, R=0.71$; Figure S2A in Supplementary Material). Afterward, we regressed GSmQTL SNPs on GS WHO_Grade (GS WHO_Grade GSmQTLs) stepwise for a minimum AIC, obtaining an adjusted $R 2$ value of 0.88 ( $P$-value $\left.<2.2 \times 10^{-16}\right)$, but indicative of multicollinearity $(\mathrm{VIF}=9.56)$. Stepwise regression by including $\mathrm{kME}$ and GSmQTLs variables (GS WHO_Grade $\sim k M E+G S m Q T L s$ ) reached an adjusted $R 2$ value of 0.89 was achieved ( $P$ value $<2.2 \times 10^{-16}$ ), but again multicollinearity was observed $(\mathrm{VIF}=10.49)$. We collected all significant variables ( $T$ test $P$ value $<0.01$ ) and divided them into two groups according to their estimates signs. The variables with negative estimates were summed into a vector known as GSmQTLProtective, whilst the variables with positive estimates were added to a vector known ad GSmQTLRisk.

GSmQTLRisk correlated strongly with GS WHO_Grade (Spearman's rho $=0.79 ; P$-value $\leq 2.2 \times 10^{-16}$; Figure S2B in Supplementary Material) while GSmQTLProtective mildly correlated with GS WHO_Grade, but that correlation was significant (Spearman's rho $=0.33 ; P$-value $=4.2 \times 10^{-14}$; Figure S2C in Supplementary Material). Modeling GS WHO_Grade with kME and these new variables instead of its originals led to a simplified model with very similar results: adjusted $R 2$ value of $0.82(P$ value $\left.<2.2 \times 10^{-16}, R=0.91\right)$. All variables ranked significant $\left(P\right.$-value $\left.<2.2 \times 10^{-16}\right)$, and multicollinearity was not observed $(\mathrm{VIF}=5.57)$. Indeed, GS WHO_Grade prediction according to this model was very accurate to reality (Spearman's rho $=0.91$, $P$-value $<2 \times 10^{-16}$; Figure S2D in Supplementary Material).

Finally, kME, GSmQTLProtective, and GSmQTL1Risk were split by their medians to dichotomize the data. After plotting the results with GS WHO_Grade, two groups of four plots each were easily differentiable, revealing that genes with higher connectivity (labeled $k+$ ) tended to have higher correlation with WHO Grade (Figure S2E in Supplementary Material). This is consistent with the finding that WHO Grade distribution is deeply correlated with connectivity. It is notorious that genes with higher values of GSmQTLProtective tended to have more negative correlations with GS WHO_Grade, while the opposite happened with genes whose GSmQTLRisk were high. The groups of genes with high $k M E$ and GSmQTLRisk and low or high GSmQTLProtective values were the most correlated with GS WHO_Stage, whilst the group of genes with low values of $k M E$ and GSmQTLRisk and high values of GSmQTLProtective was the less correlated group with GS WHO_Stage. Thus, the highest absolute correlation with meningioma Grade is achieved by genes with high connectivity and high association to putatively protective mQTLs.

\section{DISCUSSION}

Using WGCNA, we demonstrated that meningioma tissue expression can be summarized to the existence of 16 co-expression modules. One of them was found to be highly correlated with disease progression parameters. In this network, gene expression was less variable for highly connected genes than for mildly connected ones and hub genes had a higher and significant tendency to be positively correlated with tumor stage. Such characteristics are suggestive of the implication of this module in core biological functions related to tumor progression. Moreover, a group of SNPs were associated to a list of genomic regions both at the "suggestive level" (i.e., $P$-value at $10^{-5}$ level) and at the "significant level" $\left(P\right.$-value $\left.\leq 10^{-6}\right)$.

Ontology analysis of in-module genes revealed a very significant enrichment in terms related to cell-cycle pathways. Curiously, many cancer-related pathways ranked high among the results, especially isoprenoid metabolism, intermediate filament bundle assembly, proteoglycan binding, and oxidoreductase activity pathways. There is evidence pointing toward isoprenoid regulation of cell growth (52). Indeed, isoprenoid compounds are known suppressors of carcinogenic processes such as telomerase activity (53), as well as activators of cell-cycle arrest and apoptosis signals (54). Intracranial tumors are known to have an increased activity of the low-density lipoprotein receptor due to an increased requirement of cholesterol (55). In fact, there is a body of evidence suggesting that statins, which are inhibitors of the HMG-CoA reductase pathway (blocking isoprenoid synthesis), may benefit cancer therapeutics (56). Intermediate filaments are widely used as markers of cancer differentiation and prognosis $(57,58)$, while proteoglycans are known mediators of tumor cells interaction with their microenvironment and regulators of angiogenesis $(59,60)$. Oxidoreductase activity plays a role in cancer too, since a recent 
meta-analysis of 21 studies has shown that polymorphisms in the $\mathrm{NAD}(\mathrm{P}) \mathrm{H}$ quinine oxidoreductase 1 (NQO1) gene are significantly associated with digestive tract cancer risk among Europeans and Asians (61). Moreover, a careful analysis of the three network hub genes (PTTG1, PRC1, and CENPF) reveals that PTTG1 is linked to tumor malignancy and has been proposed as a possible therapeutic target (62); PRC1 encodes a factor responsible for polarizing parallel microtubules and concentrating the factors responsible for contractile ring assembly, and has been involved in the growth of breast cancer cells $(63,64)$; and that CENPF encodes a protein that associates with centromere and kinetochore complexes. CENPF amplification is a frequent event in hepatocellular carcinomas (65). These data are in concordance with previous studies where co-expression networks were found to be strongly enriched in specific functions (66-68).

A focused analysis of the top 21 hub genes revealed a marked enrichment in microtubule-associated processes, including spindle and chromosome-related pathways. Cell-cycle studies have demonstrated that the metaphase-anaphase transition depends on a correct attachment of sister chromatids to the kinetochoremicrotubule apparatus, which is by itself a crucial checkpoint (known as $M$-phase checkpoint) that prevents progression until all chromosomes are correctly joined to the spindle (69). Mad1 and Mad2 are two proteins known to regulate this checkpoint, and alterations in their ratios are known to lead to chromosome instability and aneuploidy, thus promoting tumorigenesis (70). Indeed, during anaphase, the central spindle is formed, which consists of a bundle of microtubules interpolated between segregating chromosomes and permits normal cytokinesis. Curiously, PRC1 is essential for central spindle formation, although its interactions with other spindle proteins seem tangled and await further studies (71). Thus, it is tempting to speculate that M-phase checkpoint aberrations may play an important role in meningioma malignization.

Generally speaking, the pathway and ontology results for inmodule genes are consistent with that of mQTL genes. For example, the enrichment of mQTL genes in actin and myosinrelated pathways, oxidoreductase activity, chromosome segregation, and cholesterol metabolism pathways is in accordance with co-expression module results. Nevertheless, mQTL genes were also enriched in other cancer-related pathways. For example, five mQTL putative genes are part of the cadherin family, and at least two (CHD12 and CHD13) have been broadly studied for their role in cancer (72-74). Genes related to angiogenesis and vascular permeability pathways had also significant matches, and at least one of them (NRP1) has been studied before in meningioma (49). NRP1 protein acts as a receptor of VEGF-A (75), and its blockade is under clinical research for various types to tumors (76). Indeed, the inhibition of angiogenic pathways with bevacizumab has been shown to have encouraging anti-tumoral effects in recurrent and progressing meningiomas (77). Calciumchannel activity, another significant finding in the list, is known to be involved in growth factor-mediated meningioma proliferation and the use of calcium-channel blockers has anti-proliferative and drug-sensitizing effects $(78,79)$. Two glutamatergic receptor genes were present in the mQTL list. These are GRIN2A and GRIN3A. The implication of both ionotropic and metabotropic glutamate receptors in carcinogenesis and cancer progression is well-known, especially in gliomas, melanomas, and breast and prostate cancers [reviewed in Ref. (80)]. Similarly, a region $5^{\prime}$ to SEL1L was the only duplicated gene significantly associated with meningioma progression in the two SNP cohorts. SEL1L is a tumor suppressor gene involved in the endoplasmic reticulum-associated degradation (ERAD) pathway, and known to be involved in the complex ER adaptations needed for the progression of multiple neoplasms $(81,82)$. Moreover, an SNP in the ATF6 gene was significantly associated $\left(P\right.$-value $\left.=2.65 \times 10^{-7}\right)$ with the sum of the first and second module principal components. The protein encoded by this gene is a membrane glycoprotein with a key function in the ERAD pathway and acts as a sensor and transducer of the unfolded protein response. Interestingly, it has been recently shown that misfolded ATF6 needs SEL1L for degradation despite the transmembrane nature of ATF6 (83), which indicates that degradation pathways are much more diversified than expected in eukaryotes, but with a still unknown role in cancer biology.

mQTL genes are also enriched in TFBS of the Homeobox and Forkhead family of transcription factors, and indeed FOXP1 and HOXC13 are inside the mQTL list. The implication of both families of transcription factors in cancer development and progression is widespread, and they probably play important roles in meningioma malignancy too $(84,85)$. Similarly, a marked enrichment in MIR-124A and MIR-34B microRNAs was observed. This is in accordance to the known role of both genes in cancer-related pathways. For example, MIR-124A is correlated with breast cancer growth and aggressiveness (86), whilst MIR-34B, which has tumorsuppressive effects through the inhibition of $B C L-2$ (87), is associated to renal cell cancer risk (88) and silenced in hepatocellular carcinoma (89).

The results indicate that the co-expression module as a whole is associated with a list of more than 100 SNPs genome-wide (here known as $m Q T L s$ ), with $P$-values in the range of $10^{-5}$ or less. By integrating gene-expression network connectivity and the correlation of each module gene with WHO Meningioma Grade (GS WHO_Grade) and with mQTLs (GSmQTLs), a multivariate regression model was created with only three regressors that explain almost $82 \%$ of the variability. Overall, these findings indicate that mQTLs not only are associated with the module expression, but also add a significant amount of explained variability to the study models, reinforcing their biological importance.

The main limitation facing this pipeline is related to the short sample size of the genotypic cohorts. With the aim to overcome this problem, only disease-associated gene findings present in at least two of the three SNP lists created were considered. Moreover, the consistency of the findings with gene expression, PPI networks, miRNAs and TFBS target sites, pathway and ontology databases, and current scientific literature adds plausibility and supports the veracity of the final results. However, a word of caution must be added to the results, and this applies particularly to all gene-specific findings described above, since many of them need further experimental validation. It is the aim of our study to provide a framework of evidence for future research on the field of meningiomas. 


\section{CONCLUSION}

This study has evidenced that meningioma gene expression contains a co-expression module highly correlated with tumor malignancy, whose hub genes are markedly enriched in microtubulerelated pathways. Genetic loci linked to cytoskeleton, cell-cell adhesion, angiogenesis, calcium-channels, glutamate receptors, and ERAD pathways were determined as potential regulators of the whole co-expression network. Integration of these data with alternative gene-expression data, PPI networks, TFBS and miRNA target sequence databases, and scientific literature confirmed the enrichment of mQTLs in functional cancer-related networks. Moreover, SNP association with meningioma stage evidenced that a gene of the ERAD pathway, SEL1L, is likely associated with the malignant transformation of meningiomas, consistent with mQTL, and state-of-the-art knowledge. To sum up, this paper presents for the first time an integrated view of gene expression and genomic markers in the field of meningiomas. This resulted in the discovery of several pathways involved in meningioma malignant conversion and paves the way for the development of future research lines that will shed light on meningioma biology and its treatment. The reader is encouraged to watch the video summary in Video S1 in Supplementary Material (https: //www.youtube.com/watch? $\mathrm{v}=\mathrm{fSf} 9 \mathrm{QVu} \_l \mathrm{lmE} \&$ feature=youtu.be).

\section{AUTHOR CONTRIBUTIONS}

This study was written and directed by Adrián Mosquera Orgueira. José Carlos Iglesias Gómez took part in the development of the visual content of the study and in the interpretation of the results.

\section{ACKNOWLEDGMENTS}

We would like to thank Federico Martinón-Torres [Servizo Galego de Saúde (SERGAS)], Antonio Salas Ellacuriaga (University of Santiago de Compostela), Lachlan Coin (University of Queensland), and Adonis Skandalis (Brock University) for kindly reading and making suggestions about this paper before submission. Our special thanks to Cristina and Ana Ma Gil Rey, and Aramis and Canela Mermur for their support and contributions in the writing process. José Carlos Iglesias Gómez would like to dedicate this study to his son José Carlos Iglesias Lamas (actually, "his hero"), and Adrián Mosquera dedicates it to all his family [specially both parents, both grandmothers, brother (Nicolás Mosquera)], his neighbors (e.g., Armas' bar) and specially to his recently deceased grand-parents (José Orgeira Louro and José Mosquera Bazán) whose memory light his path in a gloomy time. Thanks for the inspiration to Sir Ken Robinson for his mindful and stimulating conferences. This work has not received financial support, but we would like to thank the editors of Frontiers for the full submission waiver that allows this publication.

\section{SUPPLEMENTARY MATERIAL}

The Supplementary Material for this article can be found online at http://www.frontiersin.org/Journal/10.3389/fonc.2014. 00147/abstract

Figure S1 | (A) Boxplots showing average correlation of each module's genes and WHO Meningioma classification. (B) Plot of Module Membership for each gene and Pearson's correlation for WHO Meningioma classification. (C) Plot of pink-module gene-expression standard deviation vs. intramodular connectivity reflects that low connectivity genes are more variable.

Figure S2 | (A) Plotting of connectivity (kME) vs. GSWHO_Grade reveals a significant $\left(P\right.$-value $\left.<10^{-20}\right)$ Spearman's correlation of 0.73 between both variables. (B) Spearman's correlation of GSmQTLRisk and GSWHO_Grade is significant ( $P$-value $<10^{-20}$ ) and particularly high $(R=0.79$ ). (C) Spearman's correlation of GSmQTLProtective and GSWHO_Grade is significant $\left(P\right.$-value $\left.=4.2 \times 10^{-14}\right)$ and has a value of 0.33 . (D) Prediction of GSWHO_Grade based on a multivariate regression linear model (where KME,

GSmQTLProtective, and GSmQTLRisk were regressed on GSWHO_Grade) reveals strong and significant Spearman's correlations with observed GS WHO_Grade values. (E) Dichotomized plots by the medians of kME $(K \pm)$, GSmQTLRisk $(R \pm)$, and GSmQTLProt $(P \pm)$ evidence of two big groups based on kME values. Genes with high connectivity were more markedly correlated with GSWHO_Grade than those with lower connectivity values.

Video S1 |Artistic video summarizing the important aspects of the study Link: https://www.youtube.com/watch?v=fSf90Vu_ImE\&feature=youtu.be

Table S1 | Summary of the original data downloaded for the study, including the links to the expression and genotypic files and the phenotypic information.

Table S2 | (A) mQTL list of association between SNPs and the ME. (B) mQTL list of association between the second principal component of the co-expression module and the SNPS. The base model of this regression was calculated including the ME. (C) Similar to (B) but the regressor was the sum of the ME and the second principal component. (D) mQTL list of association between SNPs and the sum of the first and second principal components of the co-expression module. (E) miRNA target site enrichment results for mQTL putative genes. (F) Pathway and Gene Ontology enrichment of mQTL putative genes. (G) Results of TFBS enrichment in mQTL putative genes. (H) SNP association with meningioma Grade according to the discovery cohort.

Table S3 | Annotation of the pink-module probes according to the array manufacturer

Table S4 | Hub genes list and network information for each one (degree and betweenness values)

Table S5 | Cell compartment gene ontology of the network hub genes, according to DAVID.

Table S6 | Top significant results of SNP association with tumor Grade in the replication cohort.

\section{REFERENCES}

1. Santosh S, McCarthy BJ, Villano JL. Update on meningiomas. Oncologist (2011) 16:1604-13. doi:10.1634/theoncologist.2011-0193

2. Perry A, David HG, Guido R. Molecular pathogenesis of meningiomas. J Neurooncol (2004) 70(2):183-202. doi:10.1007/s11060-004-2749-0

3. Fauci AS. Harrison's Principles of Internal Medicine. New York, NY: McGraw-Hill Medical (2008).

4. Yang SY, Park CK, Park SH, Kim DG, Chung YS, Jung HW. Atypical and anaplastic meningiomas: prognostic implications of clinicopathological features. J Neurol Neurosurg Psychiatry (2008) 79(5):574-80. doi:10.1136/jnnp.2007.121582

5. Claus EB, Park PJ, Carroll R, Chan J, Black PM. Specific genes expressed in association with progesterone receptors in meningioma. Cancer Res (2008) 68(1):314-22. doi:10.1158/0008-5472

6. Kauffman SA. The Origins of Order: Self-Organization and Selection in Evolution. New York, NY: Oxford University Press (1993).

7. Dinicola S, Fabrizio D, Alessia P, Sara P, Elisabetta L, Alessandra C, et al. A systems biology approach to cancer: fractals, attractors, and nonlinear dynamics. OMICS (2011) 15(3):93-104. doi:10.1089/omi.2010.0091

8. Barabási AL, Albert R. Emergence of scaling in random networks. Science (1999) 286:509-12. doi:10.1126/science.286.5439.509 
9. Barabási AL, Bonabeau E. Scale-free networks. Sci Am (2003) 288(5):50-9. doi:10.1038/scientificamerican0503-60

10. Barabasi AL. Scale-free networks: a decade and beyond. Science (2009) 325(5939):412-3. doi:10.1126/science.1173299

11. Barabási AL, Gulbahce N, Loscalzo J. Network medicine: a network-based approach to human disease. Nat Rev Genet (2011) 12(1):56-68. doi:10.1038/ nrg2918

12. Barabási AL. Network medicine - from obesity to the "diseasome”. N Engl J Med (2007) 357(4):404-7. doi:10.1056/full/NEJMe078114

13. Goh KI, Cusick ME, Valle D, Childs B, Vidal M, Barabasi AL. The human disease network. Proc Natl Acad Sci U S A (2007) 104(21):8685-90. doi:10.1073/pnas. 0701361104

14. Liu ZP, Zhan XS, Chen L. Network-based analysis of complex diseases. IET Syst Biol (2012) 6(1):22-33. doi:10.1049/iet-syb.2010.0052

15. Langfelder P, Horvath S. WGCNA: an R package for weighted correlation network analysis. BMC Bioinformatics (2008) 9:559. doi:10.1186/1471-2105-9-559

16. Lee Y, Liu J, Pattel S, Cloughesy T, Lai A, Faroogi H, et al. Genomic landscape of meningiomas. Brain Pathol (2010) 20(4):751-62. doi:10.1111/j.1750-3639. 2009.00356.x

17. Langfelder P, Horvath S. Fast R functions for robust correlations and hierarchical clustering. J Stat Softw (2012) 46(11):1-17.

18. Ghazalpour A, Doss S, Zhang B, Wang S, Plaisier C, Castellanos R, et al. Integrating genetic and network analysis to characterize genes related to mouse weight. PLoS Genet (2005) 2(8):e130. doi:10.1371/journal.pgen.0020130

19. Edgar R, Domrachev M, Lash AE. Gene expression omnibus: NCBI gene expression and hybridization array data repository. Nucleic Acids Res (2002) 30(1):207-10. doi:10.1093/nar/30.1.207

20. Barrett T, Wilhite SE, Ledoux P, Evangelista C, Kim IF, Tomashevsky M, et al. NCBI GEO: archive for functional genomics data sets - update. Nucleic Acids Res (2013) 41:D991-5. doi:10.1093/nar/gks1193

21. R Core Team. R: A Language and Environment for Statistical Computing. Vienna: R Foundation for Statistical Computing (2012). Available from: http: //www.R-project.org/

22. Gautier L, Cope L, Bolstad BM, Irizarry RA. Affy - analysis of Affymetrix GeneChip data at the probe level. Bioinformatics (2004) 20(3):307-15. doi:10. 1093/bioinformatics/btg405

23. Kauffmann A, Gentleman R, Huber W. arrayQualityMetrics - a bioconductor package for quality assessment of microarray data. Bioinformatics (2009) 25(3):415-6. doi:10.1093/bioinformatics/btn647

24. Guy B, Van Helden J. Statistics for Bioinformatics (2013). Available from: http://www.bigre.ulb.ac.be/courses/statistics_bioinformatics/practicals/ microarrays_berry_2010/

25. Bengtsson H, Corrada-Bravo H, Gentleman R, Jaffee H. matrixStats: Methods that Apply to Rows and Columns of a Matrix. R package version 0.6.2. (2012).

26. Smoot ME, Ono K, Ruscheinski J, Wang PL, Ideker T. Cytoscape 2.8: new features for data integration and network visualization. Bioinformatics (2011) 27(3):431-2. doi:10.1093/bioinformatics/btq675

27. Scardoni G, Petterlini M, Laudanna C. Analyzing biological network parameters with CentiScaPe. Bioinformatics (2009) 25(21):2857-9. doi:10.1093/ bioinformatics/btp517

28. Bindea G, Mlecnik B, Hackl H, Charoentong P, Tosolini M, Kirilovsky A, et al. ClueGO: a cytoscape plug-in to decipher functionally grouped gene ontology and pathway annotation networks. Bioinformatics (2009) 25(8):1091-3. doi:10.1093/bioinformatics/btp101

29. The Gene Ontology Consortium. Gene ontology: tool for the unification of biology. Nat Genet (2000) 25(1):25-9. doi:10.1038/75556

30. Kanehisa M, Goto S, Sato Y, Furumichi M, Tanabe M. KEGG for integration and interpretation of large-scale molecular datasets. Nucleic Acids Res (2012) 40:D109-14. doi:10.1093/nar/gkr988

31. Kanehisa M, Goto S. KEGG: Kyoto encyclopedia of genes and genomes. Nucleic Acids Res (2000) 28:27-30. doi:10.1093/nar/28.1.27

32. Matthews L, Gopinath G, Gillespie M, Caudy M, Croft D, De Bono B, et al. Reactome knowledgebase of human biological pathways and processes. Nucleic Acids Res (2009) 37:D619-22. doi:10.1093/nar/gkn863

33. Huang DW, Sherman BT, Lempicki RA. Systematic and integrative analysis of large gene lists using DAVID bioinformatics resources. Nat Protoc (2009) 4(1):44-57. doi:10.1038/nprot.2008.211
34. Huang DW, Sherman BT, Lempicki RA. Bioinformatics enrichment tools: paths toward the comprehensive functional analysis of large gene lists. Nucleic Acids Res (2009) 37(1):1-13. doi:10.1093/nar/gkn923

35. Clayton D. snpStats: SnpMatrix and XSnpMatrix Classes and Methods. R package version 1.6.0. (2012).

36. Ward LD, Kellis M. HaploReg: a resource for exploring chromatin states, conservation, and regulatory motif alterations within sets of genetically linked variants. Nucleic Acids Res (2012) 40:D930-4. doi:10.1093/nar/gkr917

37. Ho Sui SJ, Fulton DL, Arenillas DJ, Kwon AT, Wasserman WW. oPOSSUM: integrated tools for analysis of regulatory motif over-representation. Nucleic Acids Res (2007) 35(2):W245-52. doi:10.1093/nar/gkm427

38. Subramanian A. From the cover: gene set enrichment analysis: a knowledgebased approach for interpreting genome-wide expression profiles. Proc Natl Acad Sci U S A (2005) 102(43):15545-50. doi:10.1073/pnas.0506580102

39. Franceschini A, Szklarczyk D, Frankild S, Kuhn M, Simonovic M, Roth A, et al. STRING v9.1: protein-protein interaction networks, with increased coverage and integration. Nucleic Acids Res (2013) 41:D808-15. doi:10.1093/nar/gks1094

40. Tabernero MD, Maillo A, Gil-Bellosta CJ, Castrillo A, Sousa P, Merino M, et al. Gene expression profiles of meningiomas are associated with tumor cytogenetics and patient outcome. Brain Pathol (2009) 19(3):409-20. doi:10.1002/ijc.24568

41. Stuart JE, Lusis EA, Scheck AC, Coons SW, Lal A, Perry A, et al. Identification of gene markers associated with aggressive meningioma by filtering across multiple sets of gene expression arrays. J Neuropathol Exp Neurol (2011) 70(1):1-12. doi:10.1097/NEN.0b013e3182018f1c

42. Leek JT, Johnson WE, Parker HS, Jaffe AE, Storey JD. sva: Surrogate Variable Analysis. R package version 3.2.1.

43. Smyth GK. Limma: linear models for microarray data. In: Gentleman VC, Dudoit S, Irizarry R, Huber W, editors. Bioinformatics and Computational Biology Solutions using $R$ and Bioconductor. New York, NY: Springer (2005). p. 397-420.

44. Tabernero MD, Maíllo A, Nieto AB, Diez-Tascón C, Lara M, Sousa P, et al. Delineation of commonly deleted chromosomal regions in meningiomas by highdensity single nucleotide polymorphism genotyping arrays. Genes Chromosomes Cancer (2012) 51(6):606-17. doi:10.1002/gcc.21948

45. GenABEL Project Developers. GenABEL: Genome-Wide SNP Association Analysis. R package version 1.7-4. (2013).

46. Minato Nakazawa. fmsb: Functions for Medical Statistics Book with Some Demographic Data. R package version 0.3.8. (2013).

47. Hastie T, Tibshirani R, Narasimhan B, Chu G. impute: Imputation for Microarray Data. R package version 1.30.0.

48. Mar JC, Matigian NA, Mackay-Sim A, Mellick GD, Sue CM, Silburn PA, et al. Variance of gene expression identifies altered network constraints in neurological disease. PLoS Genet (2011) 7(8):e1002207. doi:10.1371/journal.pgen. 1002207

49. Nassehi D, Sørensen LP, Dyrbye H, Thomsen C, Juhler M, Laursen H, et al. Peritumoral brain edema in angiomatous supratentorial meningiomas: an investigation of the vascular endothelial growth factor A pathway. APMIS (2013) 121(11):1025-36. doi:10.1111/apm.12052

50. Collins IM, Beddy P, O’Byrne KJ. Radiological response in an incidental meningioma in a patient treated with chemotherapy combined with CP751,871, an IGF-1R inhibitor. Acta Oncol (2010) 49(6):872-4. doi:10.3109/ 02841861003752408

51. Devlin B, Roeder K. Genomic control for association studies. Biometrics (1999) 55(4):997-1004. doi:10.1111/j.0006-341X.1999.00997.x

52. Wejde J, Carlberg M, Hjertman M, Larsson O. Isoprenoid regulation of cell growth: identification of mevalonate-labelled compounds inducing DNA synthesis in human breast cancer cells depleted of serum and mevalonate. J Cell Physiol (1993) 155(3):539-48. doi:10.1002/jcp.1041550312

53. Sundin T, Peffley DM, Gauthier D, Hentosh P. The isoprenoid perillyl alcohol inhibits telomerase activity in prostate cancer cells. Biochimie (2012) 94:2639-48. doi:10.1016/j.biochi.2012.07.028

54. Yoshikawa N, Tsuno NH, Okaji Y, Kawai K, Shuno Y, Nagawa H, et al. Isoprenoid geranylgeranylacetone inhibits human colon cancer cells through induction of apoptosis and cell cycle arrest. Anticancer Drugs (2010) 21(9):850-60. doi:10.1097/CAD.0b013e32833e53cf

55. Rudling MJ, Angelin B, Peterson CO, Collins VP. Low density lipoprotein receptor activity in human intracranial tumors and its relation to the cholesterol requirement. Cancer Res (1990) 50(3):483-7. 
56. Osmak M. Statins and cancer: current and future prospects. Cancer Lett (2012) 324:1-12. doi:10.1016/j.canlet.2012.04.011

57. Bergh J, Nilsson K, Dahl D, Andersson L, Virtanen I, Lehto VP. Expression of intermediate filaments in established human lung cancer cell lines. An indicator of differentiation and derivation. Lab Invest (1984) 3(51):307-12.

58. Bell CD, Tischler EM, Laroye GJ. The relationship of cytoplasmic intermediate filaments and membrane antigens with hormone receptors, nuclear staining density, and mode of stromal invasion in human breast cancer. Breast Cancer Res Treat (1995) 33(2):147-62. doi:10.1007/BF00682722

59. Garusi E, Rossi S, Perris R. Antithetic roles of proteoglycans in cancer. Cell Mol Life Sci (2012) 69(4):553-79. doi:10.1007/s00018-011-0816-1

60. Iozzo RV, Sanderson RD. Proteoglycans in cancer biology, tumour microenvironment and angiogenesis. J Cell Mol Med (2011) 15(5):1013-31. doi:10.1111/ j.1582-4934.2010.01236.x

61. Yang FY, Guan QK, Cui YH, Zhao ZQ, Rao W, Xi Z. NAD(P)H quinone oxidoreductase 1 (NQO1) genetic C609T polymorphism is associated with the risk of digestive tract cancer: a meta-analysis based on 21 case-control studies. Eur J Cancer Prev (2012) 21(5):432-41. doi:10.1097/CEJ.0b013e32834f7514

62. Yoon CH, Kim MJ, Lee H, Kim RK, Lim EJ, Yoo KC, et al. PTTG1 oncogene promotes tumor malignancy via epithelial to mesenchymal transition and expansion of cancer stem cell population. J Biol Chem (2012) 23(287):19516-27. doi:10.1074/jbc.M111.337428

63. Shimo A, Nishidate T, Ohta T, Fukuda M, Nakamura Y, Katagiri T. Elevated expression of protein regulator of cytokinesis 1 , involved in the growth of breast cancer cells. Cancer Sci (2007) 98(2):174-81. doi:10.1111/j.1349-7006. 2006.00381.x

64. Shrestha S, Wilmeth LJ, Eyer J, Shuster CB. PRC1 controls spindle polarization and recruitment of cytokinetic factors during monopolar cytokinesis. Mol Biol Cell (2012) 7(23):1196-207. doi:10.1091/mbc.E11-12-1008

65. Kim HE, Kim DG, Lee KJ, Son JG, Song MY, Park YM, et al. Frequent amplification of CENPF, GMNN and CDK13 genes in hepatocellular carcinomas. PLoS One (2012) 7(8):e43223. doi:10.1371/journal.pone.0043223

66. Horvath S, Zhang B, Carlson M, Lu KV, Zhu S, Felciano RM, et al. Analysis of oncogenic signaling networks in glioblastoma identifies ASPM as a molecular target. Proc Natl Acad Sci U S A (2006) 103(46):17402-7. doi:10.1073/pnas. 0608396103

67. Oldham MC, Konopka G, Iwamoto K, Langfelder P, Kato T, Horvath S, et al. Functional organization of the transcriptome in human brain. Nat Neurosci (2008) 11(11):1271-82. doi:10.1038/nn.2207

68. Mason MJ, Fan G, Plath K, Zhou Q, Horvath S. Signed weighted gene coexpression network analysis of transcriptional regulation in murine embryonic stem cells. BMC Genomics (2009) 10(1):327. doi:10.1186/1471-2164-10-327

69. Lara-Gonzalez P, Westhorpe FG, Taylor SS. The spindle assembly checkpoint. Curr Biol (2012) 22(22):R966-80. doi:10.1016/j.cub.2012.10.006

70. Schuyler SC, Wu YF, Kuan VJ. The Mad1-Mad2 balancing act - a damaged spindle checkpoint in chromosome instability and cancer. J Cell Sci (2012) 125(18):4197-206. doi:10.1242/jcs.107037

71. Lee KY, Davies T, Mishima M. Cytokinesis microtubule organisers at a glance. J Cell Sci (2012) 125(15):3495-500. doi:10.1242/jcs.094672

72. Wang JF, She L, Su BH, Ding LC, Zheng FF, Zheng DL, et al. CDH12 promotes the invasion of salivary adenoid cystic carcinoma. Oncol Rep (2011) 1:101-8. doi:10.3892/or.2011.1286

73. Lin YL, He ZK, Li ZG, Guan TY. Downregulation of CDH13 expression promotes invasiveness of bladder transitional cell carcinoma. Urol Int (2013) 90(2):225-32. doi:10.1159/000345054

74. Wang Z, Yuan X, Jiao N, Zhu H, Zhang Y, Tong J. CDH13 and FLBN3 gene methylation are associated with poor prognosis in colorectal cancer. Pathol Oncol Res (2012) 18(2):263-70. doi:10.1007/s12253-011-9437-0

75. Plein A, Fantin A, Ruhrberg C. Neuropilin regulation of angiogenesis, arteriogenesis, and vascultar permeability. Microcirculation (2014) 21(4):315-23. doi:10.1111/micc.12124

76. Patnaik A, Lorusso PM, Messersmith WA, Papadopoulos KP, Gore L, Beeram M, et al. A Phase Ib study evaluating MNRP1685A, a fully human anti-NRP1 monoclonal antibody, in combination with bevacizumab and paclitaxel in patients with advanced solid tumors. Cancer Chemother Pharmacol (2014) 73(5):951-60. doi:10.1007/s00280-014-2426-8

77. Lou E, Sumrall AL, Turner S, Peters KB, Desjardins A, Vredenburgh JJ, et al. Bevacizumab therapy for adults with recurrent/progressive meningioma: a retrospective series. J Neurooncol (2012) 109(1):63-70. doi:10.1007/s11060-012-0861-0

78. Jensen RL, Petr M, Wurster RD. Calcium channel antagonist effect on in vitro meningioma signal transduction pathways after growth factor stimulation. $\mathrm{Neu}$ rosurgery (2000) 46(3):692-702. doi:10.1097/00006123-200003000-00032

79. Ragel BT, Couldwell WT, Wurster RD, Jensen RL. Chronic suppressive therapy with calcium channel antagonists for refractory meningiomas. Neurosurg Focus (2007) 23(4):E10. doi:10.3171/FOC-07/10/E10

80. Willard SS, Koochekpour S. Glutamate signaling in benign and malignant disorders: current status, future perspectives, and therapeutic implications. Int J Biol Sci (2013) 9(7):728-42. doi:10.7150/ijbs.6475

81. Cattaneo M, Lotti LV, Martino S, Alessio M, Conti A, Bachi A, et al. Secretion of novel SEL1L endogenous variants is promoted by ER stress/UPR via endosomes and shed vesicles in human cancer cells. PLoS One (2011) 6(2):e17206. doi:10.1371/journal.pone.0017206

82. Ashktorab H, Green W, Finzi G, Sessa F, Nouraie M, Lee EL, et al. SEL1L, an UPR response protein, a potential marker of colonic cell transformation. Dig Dis Sci (2012) 57(4):905-12. doi:10.1007/s10620-011-2026-y

83. Horimoto S, Ninagawa S, Okada T, Koba H, Sugimoto T, Kamiya Y, et al. The unfolded protein response transducer ATF6 represents a novel transmembranetype endoplasmic reticulum-associated degradation substrate requiring both mannose trimming and SEL1L protein. J Biol Chem (2013) 288(44):31517-27. doi:10.1074/jbc.M113.476010

84. Katoh M, Igarashi M, Fukuda H, Nakagama H, Katoh M. Cancer genetics and genomics of human FOX family genes. Cancer Lett (2013) 328(2):198-206. doi:10.1016/j.canlet.2012.09.017

85. Haria D, Naora H. Homeobox gene deregulation: impact on the hallmarks of cancer. Cancer Hallm (2013) 1(2-3):67-76. doi:10.1166/ch.2013.1007

86. Ben Gacem R, Ben Abdelkrim O, Ziadi S, Ben Dhiab M, Trimeche M. Methylation of miR-124a-1, miR-124a-2, and miR-124a-3 genes correlates with aggressive and advanced breast cancer disease. Tumour Biol (2013) 35(5):4047-56 doi:10.1007/s13277-013-1530-4

87. Xie YL, Yang YJ, Tang C, Sheng HJ, Jiang Y, Han K, et al. Estrogen combined with progesterone decreases cell proliferation and inhibits the expression of Bcl-2 via microRNA let-7a and miR-34b in ovarian cancer cells. Clin Transl Oncol (2014). doi:10.1007/s12094-014-1166- $\mathrm{x}$

88. Zhang S, Qian J, Cao Q, Li P, Wang M, Wang J, et al. A potentially functional polymorphism in the promoter region of $\mathrm{miR}-34 \mathrm{~b} / \mathrm{c}$ is associated with renal cell cancer risk in a Chinese population. Mutagenesis (2014) 29(2):149-54. doi:10.1093/mutage/geu001

89. Xie K, Liu J, Chen J, Dong J, Ma H, Liu Y, et al. Methylation-associated silencing of microRNA-34b in hepatocellular carcinoma cancer. Gene (2014) 543(1):101-7. doi:10.1016/j.gene.2014.03.059

Conflict of Interest Statement: The authors declare that the research was conducted in the absence of any commercial or financial relationships that could be construed as a potential conflict of interest.

Received: 27 December 2013; accepted: 28 May 2014; published online: 23 June 2014. Citation: Iglesias Gómez JC and Mosquera Orgueira A (2014) An integrative analysis of meningioma tumors reveals the determinant genes and pathways of malignant transformation. Front. Oncol. 4:147. doi: 10.3389/fonc.2014.00147

This article was submitted to Cancer Genetics, a section of the journal Frontiers in Oncology.

Copyright (๑ 2014 Iglesias Gómez and Mosquera Orgueira. This is an open-access article distributed under the terms of the Creative Commons Attribution License (CC $B Y$ ). The use, distribution or reproduction in other forums is permitted, provided the original author(s) or licensor are credited and that the original publication in this journal is cited, in accordance with accepted academic practice. No use, distribution or reproduction is permitted which does not comply with these terms. 